\title{
Diffusible component from the spore surface of the fungus Aspergillus fumigatus which inhibits the macrophage oxidative burst is distinct from gliotoxin and other hyphal toxins
}

\author{
Colin G Mitchell, Joan Slight, Kenneth Donaldson
}

\begin{abstract}
Background - The fungus Aspergillus fumigatus, whose spores are present ubiquitously in the air, causes a range of diseases in the human lung. A small molecular weight $(<10 \mathrm{kD})$ heat stable toxin released from the spores of clinical and environmental isolates of $A$ fumigatus within minutes of deposition in aqueous solution has previously been described. A key effect of the toxin was to inhibit the oxidative burst of macrophages as measured by superoxide anion release. It was hypothesised that the toxin was one of the commonly found $A$ fumigatus hyphal toxins such as gliotoxin. This inhibitor may be an important factor which allows the fungus to colonise the lung.

Methods - The spore derived inhibitor was shown to inhibit the respiratory burst of rat alveolar macrophages, as measured by the generation of superoxide anion. Samples of the spore diffusate were subject to reversed phase high performance liquid chromatography (HPLC), thin layer chromatography (TLC), high performance thin layer chromatography (HPTLC), or organic extraction followed by TLC or HPLC to identify the presence of gliotoxin, fumagillin, helvolic acid, fumigaclavine- $\mathrm{C}$, and aurasperone-C. Commercially obtained preparations of the toxins gliotoxin, fumagillin and helvolic acid and extracts enriched for fumigaclavine- $C$ and aurasperone- $C$ were used as internal and external standards and in the respiratory burst measurements.
\end{abstract}

Results - Gliotoxin, fumagillin, helvolic acid, fumigaclavine- $\mathrm{C}$, and aurasperone$C$ were not detected in spore derived diffusate using HPLC or TLC. Using extraction procedures with solvents known to extract gliotoxin from A fumigatus culture supernatants, no gliotoxin was detected in the spore derived diffusate. Commercial gliotoxin, fumagillin, and helvolic acid or extracts enriched for fumigaclavine- $\mathrm{C}$ and aurasperone-C did not inhibit the oxidative burst of macrophages.

Conclusions - The hypothesis that the spore derived toxin is one of the toxins derived from hyphae such as gliotoxin, helvolic acid, fumagillin, fumigaclavine-
$C$, or aurasperone-C is not proved. The spore toxin may exert its effect through its ability to diffuse rapidly into the lung lining fluid, diminish the macrophage oxidative burst, and play a part in allowing $A$ fumigatus to persist in the lung and manifest its well known pathogenic effects.

(Thorax 1997;52:796-801)

Keywords: Aspergillus fumigatus, macrophage, gliotoxin, fumagillin, helvolic acid, fumigaclavine-C, aurasperone-C.

The fungus Aspergillus fumigatus is commonly found in the environment, its usual habitat being dead or decaying organic matter. The fungus causes many human lung diseases ${ }^{12}$ and a major factor in determining the pathogenicity of the fungus is the size of the spores (around $3 \mu \mathrm{m}$ in diameter) which are present ubiquitously in the air. Mullins et $a l^{\beta}$ have shown that many more spores of $A$ fumigatus are present in the lungs at necropsy than would be anticipated from their presence in the air. The spores therefore appear to have some survival advantage in the human lung over other spores. The survival rate of spores of $A$ fumigatus in rabbit lung is greater than that of control spores of a similar size. ${ }^{4}$ Several factors have been suggested as contributing to the pathogenesis of $A$ fumigatus.

Proteases, including a $32 \mathrm{kD}$ chymotrypsinlike protease present on spores and hyphae, have been shown to reduce cell spreading ${ }^{5}$ and promote detachment of cultured cells ${ }^{67}$ A low molecular weight component from the spore surface has been shown to inhibit the spreading and chemotaxis of macrophages, ${ }^{5}$ to inhibit the macrophage respiratory burst, ${ }^{8}$ and also to prevent phagocytosis. ${ }^{9}$ We have recently confirmed the ability of the spore derived inhibitor to inhibit the macrophage respiratory burst and characterised it as having a molecular weight of $10 \mathrm{kD}$ and to contain a carbohydrate component. ${ }^{10}$ The diffusible component can also inhibit macrophage cytokine gene expression by inhibiting transcription factor activation. ${ }^{11}$ Aspergillus hyphae are know to produce a range of well characterised toxins and we hypothesised that the spore derived inhibitor was one of these. The study enabled us to reject this hypothesis by showing that the toxin is not one of the common immunomodulating fungal hyphae toxins such as gliotoxin but is a novel one and we are currently investigating its structure. 


\section{Methods}

MATERIALS

Oxidised gliotoxin, helvolic acid, and fumagillin were from Sigma (Poole, Dorset, UK) and reduced gliotoxin was prepared by the method of Waring et al. ${ }^{12}$ All solvents were HPLC grade. In house bred adult Wistar rats were used throughout.

BRONCHOALVEOLAR LAVAGE

Rats were killed by intraperitoneal injection of Euthatal (Rhone Merieux Ltd). The lungs were dissected free of the thoracic cavity and lavaged with $4 \times 8 \mathrm{ml}$ sequential volumes of saline at $37^{\circ} \mathrm{C}$ to obtain the alveolar macrophages which were $>95 \%$ pure, the remainder being lymphocytes.

FUNGAL SPORES AND $A$ FUMIGATUS DIFFUSATE A single strain of $A$ fumigatus was isolated from the sputum of a patient with allergic bronchopulmonary aspergillosis. Spores were obtained from cultures that were grown for 10 days at $30^{\circ} \mathrm{C}$ on malt agar. Spore suspensions were prepared in Hank's balanced salt solution (HBSS, Gibco) by gentle homogenisation. Spore counts were performed using an improved Neubauer chamber. To obtain diffusate the spores were incubated at a concentration of $10^{8} / \mathrm{ml}$ in $\mathrm{HBSS}$ at $37^{\circ} \mathrm{C}$ for one hour in an orbital incubator. The soluble diffusate was passed through a Whatman number 1 filter paper followed by a $0.22 \mu \mathrm{m}$ sterile filter and stored at $-20^{\circ} \mathrm{C}$.

SUPEROXIDE ANION ASSAY

Superoxide anion was measured as previously described. ${ }^{13}$ Briefly, the assay mixture comprised $1.5 \mathrm{ml} \mathrm{HBSS}$ containing $2 \mathrm{mg} / \mathrm{ml} \mathrm{dex-}$ trose (Sigma) and $1 \mathrm{mg} / \mathrm{ml}$ cytochrome C (Sigma). Gliotoxin, fumagillin, and helvolic acid (Sigma) were dissolved in ethanol at a concentration of $1 \mathrm{mg} / \mathrm{ml}$ and stored at $-20^{\circ} \mathrm{C}$. Each toxin was added to the assay in 10-fold dilutions to give final concentrations of $1 \mathrm{ng} /$ $\mathrm{ml}$ to $10 \mu \mathrm{g} / \mathrm{ml}$. In a fourth series of experiments gliotoxin, fumagillin, and helvolic acid were added in paired combinations and also as a triplicate to see if more than one toxin had to be present to provoke an inhibitory response. In a final series of experiments spore diffusate which had been extracted with chloroform or dichloromethane was added to give a final concentration of extraction products that would be found in an equivalent one quarter dilution of the unfractionated diffusate. Bronchoalveolar macrophages $\left(0.25 \times 10^{6}\right)$ were added to each assay tube and triggered with phorbol myristate acetate (PMA) (final concentration $1 \mu \mathrm{g} / \mathrm{ml}$; Sigma). Superoxide dismutase (SOD) controls (final concentration $38 \mu \mathrm{g} / \mathrm{ml}$; Sigma) were included in each assay. All assays were performed in triplicate and incubated at $37^{\circ} \mathrm{C}$ for one hour. The supernatants were then read at $550 \mathrm{~nm}$ and $468 \mathrm{~nm}$ as previously described ${ }^{10}$ and expressed as nanomoles of superoxide anion produced $/ 10^{6}$ cells. Experiments were also carried out to assess the effect of $0.01 \%$ $(\mathrm{v} / \mathrm{v})$ ethanol which was present in the highest concentration of each toxin.

\section{VIABILITY}

To determine whether gliotoxin, fumagillin, and helvolic acid were lethal to cells, $4 \times 10^{5}$ alveolar macrophages were incubated in $10 \mu \mathrm{g} /$ $\mathrm{ml}$ of each toxin for one hour at $37^{\circ} \mathrm{C}$. Following this incubation the percentage viability was determined by the trypan blue exclusion method.

\section{IDENTIFICATION OF GLIOTOXIN BY THIN LAYER} CHROMATOGRAPHY (TLC)

Analysis of $A$ fumigatus diffusate by TLC to identify the presence of both oxidised and reduced gliotoxin was by a modification of the method of Richard et al. ${ }^{14}$ Samples $(10-20 \mu \mathrm{l})$ were spotted onto a $\mathrm{K} 6 \mathrm{~F}$ silica-60 plate (Whatman, Cat. No 4861-820), including standards of oxidised and reduced gliotoxin (5-10 $\mu \mathrm{g}$ of standard per spot). The $A$ fumigatus diffusate was concentrated 50 -fold by freeze drying before analysis. The plate was developed $14 \mathrm{~cm}$ with each of the following solvents: solvent 1 , dichloromethane:methanol $(97: 3 \mathrm{v} / \mathrm{v})$; solvent 2, chloroform:methanol $(70: 30 \mathrm{v} / \mathrm{v})$. Plates were air dried with the gliotoxin visualised as orange/brown spots using a spray containing $5 \%(\mathrm{w} / \mathrm{v})$ silver nitrate in ethanol: $\mathrm{H}_{2} \mathrm{O}(95: 5$ $\mathrm{v} / \mathrm{v})$.

\section{EXTRACTION OF DIFFUSATE WITH ORGANIC} SOLVENTS

A fumigatus diffusate $(100 \mathrm{ml})$ was extracted with $25 \mathrm{ml}$ of chloroform, ethyl acetate, or dichloromethane with shaking overnight. The organic phase was removed, evaporated to dryness under vacuum, and re-dissolved in $100 \mu \mathrm{l}$ of either chloroform or dichloromethane for TLC analysis, re-dissolved in $500 \mu \mathrm{l}$ of methanol: $\mathrm{H}_{2} \mathrm{O}(50: 50 \mathrm{v} / \mathrm{v})$ for high performance liquid chromatography (HPLC) analysis or $1 \mathrm{ml}$ of HBSS for the superoxide anion assay.

HIGH PERFORMANCE LIQUID CHROMATOGRAPHY A modification of the method of Richard et $a l^{14}$ was used to analyse $A$ fumigatus diffusate for the presence of oxidised and reduced gliotoxin. The HPLC was from LKB-Pharmacia fitted with a Spherisorb Spheri-5 RP-18 reversed phase column $(220 \times 2.1 \mathrm{~mm}, 5 \mu \mathrm{m})$ with an RP-18 Newguard $(15 \times 3.2 \mathrm{~mm}, 7 \mu \mathrm{m})$ guard column. The mobile phase was methanol: $\mathrm{H}_{2} \mathrm{O}$ $(50: 50 \mathrm{v} / \mathrm{v})$. Samples were dissolved in the mobile phase and $20 \mu \mathrm{l}$ injected onto the column at a flow rate of $0.1 \mathrm{ml} / \mathrm{min}$ with a detection wavelength of $275 \mathrm{~nm}$. All samples and solvents were filtered through a $0.2 \mu \mathrm{m}$ filter before use.

EXTRACTION AND ANALYSIS OF AURASPERONE-C This was carried out by a modification of the method of Ehrlich et al. ${ }^{15}$ In $100 \mathrm{ml}$ of di- 
chloromethane, $0.5 \mathrm{~g}$ of spores were soaked for 16 hours. The spores were removed by filtration and the dichloromethane removed by rotary evaporation. The residue was resuspended in nine volumes (vol/wt) of cold hexane and kept at $4^{\circ} \mathrm{C}$ overnight. The precipitate was filtered off and resuspended in dichloromethane. HPTLC was carried out on plates (LHP-KF; $10 \times 10 \mathrm{~cm})$ and developed with benzene:ethyl acetate:formic acid (10:4:1). Components were identified by colour after staining with the Gibbs reagent ${ }^{16}$ and by fluorescence under long wavelength ultraviolet light. Aurasperone-C has an $\mathrm{Rf}$ of around 0.49. Commercial standards of aurasperone-C are not available, so the above procedure was also carried out using the fungal mycelium. This is a rich source of naphtho- $\gamma$-pyrones and a total of eight were observed after HPTLC, including a spot at $\mathrm{Rf}=0.49$ corresponding to aurasperone-C.

\section{EXTRACTION AND ANALYSIS OF \\ FUMIGACLAVINE-C}

This was carried out according to the procedure of Cole et al. ${ }^{17}$ Briefly, a spore suspension was heated with an equal volume of chloroform and filtered through cheesecloth and through anhydrous sodium sulphate. The chloroform was removed and the precipitate resuspended in ethyl acetate. This was partitioned three times in water adjusted to $\mathrm{pH} 2.0$ with $\mathrm{HCl}$. This was adjusted to $\mathrm{pH} 10.0$ with sodium carbonate and partitioned three times with chloroform. The extract was dried over sodium carbonate and concentrated under vacuum. A $2.5 \times 10 \mathrm{~cm}$ neutral alumina column (deactivated to activity grade IV) was equilibrated in benzene. The concentrate was loaded onto the column and eluted with benzene. HPTLC was carried out on plates (LHP-KF; $10 \times 10 \mathrm{~cm})$ and developed in toluene:ethyl acetate:formic acid (5:4:1). Visualisation was by spraying with $50 \%$ ethanolic $\mathrm{H}_{2} \mathrm{SO}_{4}$ and heating for five minutes at $100^{\circ} \mathrm{C}$. Fumigaclavine-C was observed as a blue spot with $\mathrm{Rf}=0.14$. Commercial standards of fumigaclavine- $\mathrm{C}$ are not available, so the above procedure was also carried out using the fungal mycelium. This is a rich source of clavine alkaloids and a spot at $\mathrm{Rf}=0.14$ corresponding to fumigaclavine- $\mathrm{C}$ was observed.

\section{EXPERIMENTAL DESIGN AND STATISTICAL} ANALYSIS

Superoxide experiments were carried out in triplicate and performed on three or four separate occasions. The raw data were subjected to analysis of variance with the Tukey test for significance of differences between treatments with $\mathrm{p}<0.05$ taken as the level of significance.

\section{Results}

ANALYSIS OF DIFFUSATE BY TLC

Oxidised gliotoxin

In the solvent system dichloromethane:methanol $(97: 3 \mathrm{v} / \mathrm{v})$ oxidised gliotoxin had an $\mathrm{Rf}$ of
Table 1 Thin layer chromatography of oxidised and reduced gliotoxin, unfractionated A fumigatus diffusate and $A$ fumigatus diffusate after organic extraction

\begin{tabular}{lll}
\hline Sample & Solvent $^{*}$ & $R f$ \\
\hline Oxidised gliotoxin & 1 & 0.61 \\
& 2 & 0.97 \\
Reduced gliotoxin & 2 & 0.93 \\
Unfractionated AfD & 1 & 0.05 \\
& 2 & NS \\
Unfractionated AfD & 1 & 0.61 \\
(+ oxidised gliotoxin) & 2 & 0.97 \\
Unfractionated AfD & 2 & 0.93 \\
(+reduced gliotoxin) & & \\
Chloroform extract of AfD & 1 & $0.48,0.45$, \\
& & $0.19,0.08$, \\
& 2 & 0.79 \\
Ethyl acetate extract of AfD & 1 & NS
\end{tabular}

$\mathrm{AfD}=$ Aspergillus fumigatus diffusate; $\mathrm{NS}=$ no spots detected. * Solvent 1 , dichloromethane:methanol $(97: 3 \mathrm{v} / \mathrm{v})$; solvent 2 , chloroform:methanol $(70: 30 \mathrm{v} / \mathrm{v})$.

0.61. Unfractionated $A$ fumigatus diffusate did not show any spots corresponding to oxidised gliotoxin although a faint spot of low mobility $(\mathrm{Rf}=0.05)$ was seen. In the solvent chloroform: methanol $(70: 30 \mathrm{v} / \mathrm{v})$ oxidised gliotoxin gave an $\mathrm{Rf}$ of 0.97 while unfractionated $A$ fumigatus diffusate showed no spots.

\section{Reduced gliotoxin}

In the solvent system dichloromethane:methanol $(97: 3 \mathrm{v} / \mathrm{v})$ standard reduced gliotoxin did not move from the origin. In the solvent chloroform:methanol $(70: 30 \mathrm{v} / \mathrm{v})$ reduced gliotoxin gave an $\mathrm{Rf}$ of 0.93 . Unfractionated $A$ fumigatus diffusate showed no spots corresponding to reduced gliotoxin, all the material remaining at the origin.

\section{Solvent extraction}

After extraction of $A$ fumigatus diffusate with ethyl acetate and development in the solvent systems dichloromethane:methanol $(97: 3 \mathrm{v} / \mathrm{v})$ and chloroform:methanol $(70: 30 \mathrm{v} / \mathrm{v})$, no spots corresponding to oxidised or reduced gliotoxin were observed. After extraction of $A$ fumigatus diffusate with chloroform, four major spots were seen at $\mathrm{Rf} 0.48,0.45,0.19$, and 0.08 (there were also three minor spots at $\mathrm{Rf}<0.08$ ). Both these extraction procedures have been used to extract gliotoxin from cultures of $A$ fumigatus $^{14}$ but, in the current study, there was no evidence for the existence of oxidised or reduced gliotoxin in $A$ fumigatus diffusate. The data for TLC analysis are given in table 1 .

\section{ANALYSIS OF DIFFUSATE BY HPLC}

Oxidised gliotoxin

Figure 1 shows the HPLC analysis of $A$ fumigatus diffusate with standard oxidised and reduced gliotoxin. Oxidised gliotoxin has a retention time of 23.5 minutes. There is no corresponding peak at this retention time after HPLC of $A$ fumigatus diffusate. This suggests that there is no oxidised gliotoxin in A fumigatus diffusate. 


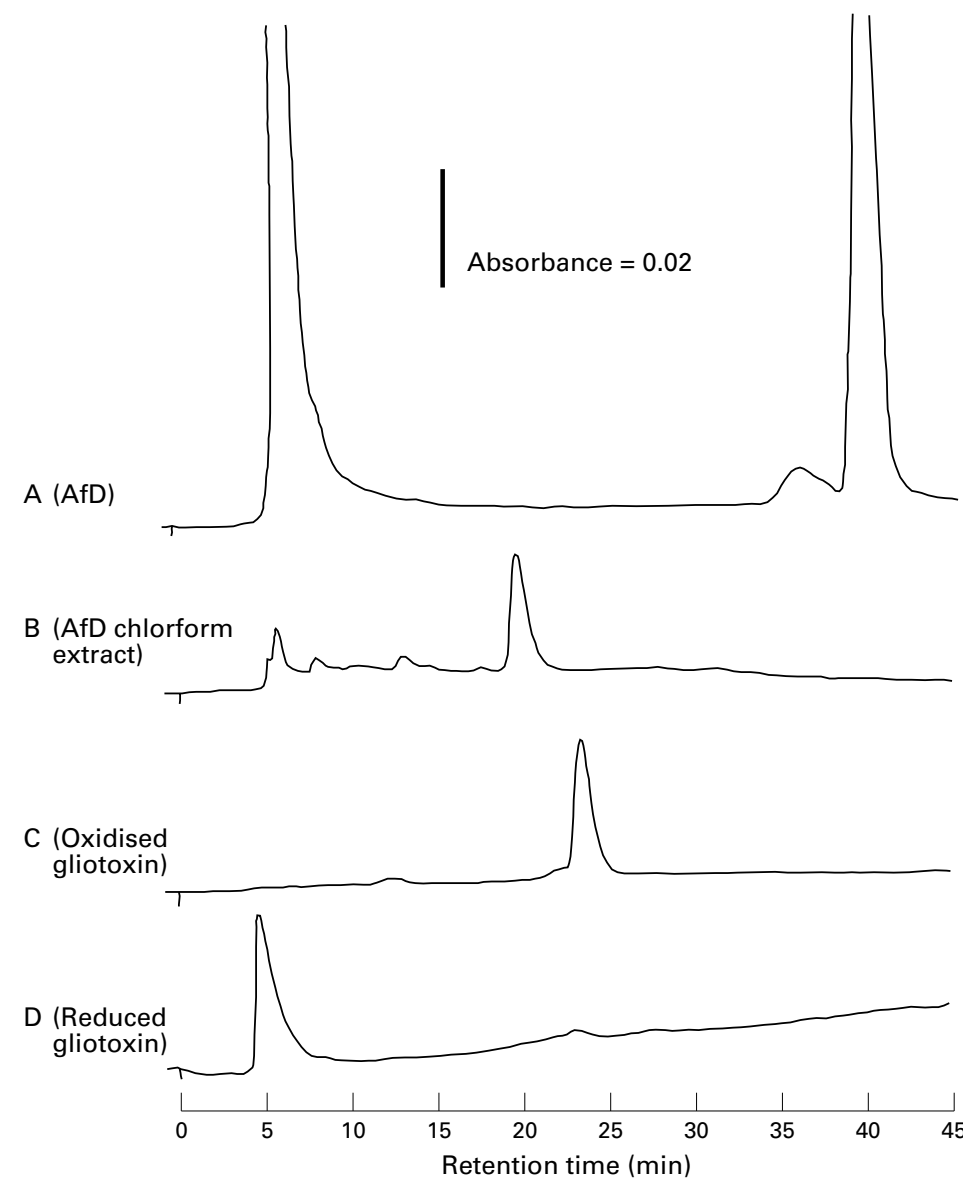

Figure 1 HPLC analysis of A fumigatus diffusate (AfD). The following traces are shown: (A) unfractionated AfD; (B) chloroform extract of AfD; (C) oxidised gliotoxin; (D) reduced gliotoxin.
Table 2 The effect of gliotoxin, fumagillin and helvolic acid on superoxide anion production

\begin{tabular}{ccll}
\hline Treatment & Gliotoxin & Fumagillin & Helvolic acid \\
\hline None & $70.3(8.8)$ & $39.7(1.5)$ & $66.3(12.0)$ \\
$1 \mathrm{ng}$ & $88.0(9.0)$ & $39.9(3.9)$ & $61.9(9.8)$ \\
$10 \mathrm{ng}$ & $75.4(8.7)$ & $39.8(5.3)$ & $64.1(10.3)$ \\
$100 \mathrm{ng}$ & $117.3(10.8) \dagger$ & $41.2(1.2)$ & $64.3(10.5)$ \\
$1 \mu \mathrm{g}$ & $89.3(10.9)$ & $39.5(1.8)$ & $61.0(9.8)$ \\
$10 \mu \mathrm{g}$ & $72.6(10.7)$ & $16.8(1.0)^{*}$ & $22.1(5.7)^{*}$ \\
\hline
\end{tabular}

Data shown are expressed as nmoles of superoxide produced $10^{6}$ cells and are the mean (SE) of three (fumagillin, helvolic acid) or four experiments (gliotoxin).

* Significant decrease $(p<0.05)$ from none.

† Significant increase $(\mathrm{p}<0.05)$ from none.

Table 3 Effect of A fumigatus diffusate (AfD) and organic extracts of spores on superoxide anion production collected under conditions that would enrich for fumigaclavine- $C$ (chloroform extract) or aurasperone- $C$ (dichloromethane extract)

\begin{tabular}{llll}
\hline Treatment & AfD & $\begin{array}{l}\text { Spore extract } \\
\text { enriched for } \\
\text { fumigaclavine- } C\end{array}$ & $\begin{array}{l}\text { Spore extract } \\
\text { enriched for } \\
\text { aurasperone- } C\end{array}$ \\
\hline None & $87.4(5.6)$ & $93.2(2.9)$ & $89.4(6.3)$ \\
1/4 dilution & $27.3(6.4)$ & $88.5(6.3)$ & $92.4(5.5)$ \\
\hline
\end{tabular}

Data shown are expressed as nmoles of superoxide produced/ $10^{6}$ cells and are the mean (SE) of three experiments.

SUPEROXIDE ANION ASSAY

Gliotoxin showed no inhibitory effect at any dilution on superoxide anion production by rat alveolar macrophages. A significant stimulation was, however, detected at $100 \mathrm{ng} / \mathrm{ml}$ (table 2). Both fumagillin and helvolic acid showed a significant decrease in superoxide production only at the highest dose of $10 \mu \mathrm{g} / \mathrm{ml}$. On assaying $0.01 \%(\mathrm{v} / \mathrm{v})$ ethanol alone, the concentration present in the $10 \mu \mathrm{g} / \mathrm{ml}$ dilution, it was found to inhibit superoxide production by $74 \%$, thus explaining the decrease found only at the highest concentration of both fumagillin and helvolic acid. This effect was not found with gliotoxin, suggesting that it may offer some protection from the effects of ethanol. When gliotoxin, fumagillin, and helvolic acid were added in paired combinations or as mixtures of all three, no inhibition of superoxide anion was observed. All combinations where gliotoxin was present showed a significant increase $(\mathrm{p}<0.05)$ in superoxide anion production (data not shown), as found in the previous experiments. There was no inhibitory effect of the unfractionated chloroform or dichloromethane spore extracts enriched for fumigaclavine-C or aurasperone-C, respectively (table 3 ). lar to reduced gliotoxin. However, this is unlikely to be reduced gliotoxin since none was detected in the $A$ fumigatus diffusate by TLC. No peaks were seen for $A$ fumigatus diffusate extracted with ethyl acetate (results not shown) which is again in agreement with TLC analysis.

Fumagillin, fumigaclavine- $C$, aurasperone- $C$ A single peak with a retention time of 12.0 minutes was seen after HPLC for standard fumagillin (data not shown), with no corresponding peak in the unfractionated $A f u$ migatus diffusate or in the chloroform extract. Neither aurasperone-C nor fumigaclavine-C were found in the spore diffusate by HPTLC, but both were present in the mycelial extracts (data not shown).

\section{VIABILITY}

When macrophages were treated with $10 \mu \mathrm{g} / \mathrm{ml}$ of commercially obtained gliotoxin, fumagillin, and helvolic acid there was no evidence of lethality or loss of membrane integrity compared with untreated macrophages (data not shown), as shown by trypan blue exclusion.

\section{Discussion}

Reports on the effect of the $A$ fumigatus spore diffusate on inhibition of phagocyte migration, spreading, and the oxidative burst have been numerous. ${ }^{5-7}$ In addition, the hyphae of $A$ fumigatus release an inhibitor of the com- 
plement system, the other key defence system of the alveolar region of the lung. ${ }^{18}$ Previous studies have demonstrated the presence of an inhibitor of the macrophage oxidative burst that is present on all $A$ fumigatus spores studied and is released from the surface of the spore. ${ }^{610}$ The toxin is removed rapidly from the spore surface (within two minutes), is heat stable to $100^{\circ} \mathrm{C}$, is of low molecular weight $(<10 \mathrm{kD})$, and appears to be associated with a carbohydrate component of the diffusate. ${ }^{10}$

The hyphae of $A$ fumigatus have been found to release a number of other low molecular weight toxins - specifically gliotoxin, helvolic acid, and fumagillin ${ }^{19}$ - and it has been reported that the toxins fumigaclavine- $\mathrm{C}$ and aurasperone-C are also associated with the fungal conidia. ${ }^{20}$ Hyphal gliotoxin ${ }^{21}$ is believed to be an important factor in allowing the hyphae to grow in tissue. Gliotoxin is able dramatically to modulate lung cell functions such as attachment of epithelial cells and fibroblasts as well as inhibiting phagocytosis by macrophages; other important functions of the host immune defence are also impaired by gliotoxin, including induction of cytotoxic and alloreactive $\mathrm{T}$ cells. ${ }^{22}$ Aurasperone-C, fumigaclavine-C, helvolic acid, and fumagillin have not been reported to have direct toxic effects on macrophages, but they do elicit severe clinical effects. Aurasperone- $\mathrm{C}$ is a tremerogen causing nervous system dysfunction. ${ }^{17}$ Fumigaclavine-C is an ergot alkaloid normally produced by $\mathrm{Cla}$ viceps species but is also present in A fumigatus $^{23}$ and causes nausea, vomiting, delirium, and coma. Helvolic acid may cause fatty degeneration of the liver, while fumagillin is a potent inhibitor of DNA synthesis. ${ }^{24}$

We have previously reported that the spore diffusate of $A$ fumigatus inhibits the macrophage oxidative burst ${ }^{10}$ and cytokine gene expression. ${ }^{11}$ In this paper we have eliminated the possibility that the spore toxin is one of the common Aspergillus toxins, particularly the potent immunomodulator gliotoxin. Gliotoxin is reported not to be present in cultures of $A$ fumigatus until three days ${ }^{25} 26$ which suggests that it is a hyphal, rather than a spore, product. However, the spores and hyphae of $A$ fumigatus have been shown to have a number of antigens in common, as has been demonstrated by the use of monoclonal antibodies. ${ }^{27}$ Isolation of gliotoxin is carried out by chloroform extraction of culture supernatants after the removal of the fungal mycelium..$^{212526}$ Further fractionation of chloroform extracts showed that ethyl acetate was a useful solvent for extracting gliotoxin. ${ }^{2}$ Maximal yield of gliotoxin was produced in shaking liquid cultures with little or none being produced in static cultures, since in static culture the fungus was shown to be sporulating abundantly. Gliotoxin production seems therefore not to be associated with spore production. ${ }^{12}$ Only the oxidised form of gliotoxin has been isolated from these cultures and the reduced form synthesised chemically in vitro. As far as the authors are aware, there have been no reports cited in the literature on the isolation of reduced gliotoxin from Aspergillus species. The data presented in this paper show that none of the toxins gliotoxin, helvolic acid, fumagillin, fumigaclavine- $\mathrm{C}$, and aurasperone- $\mathrm{C}$ are found in the spore diffusate, neither do commercial preparations or organic extracts (which should contain the toxins if present) of spores or hyphae inhibit the macrophage oxidative burst. It appears, therefore, that the immunomodulating effects of the spore diffusate, including inactivation of the cytokine transcription factor $\mathrm{NF}-\kappa \mathrm{B}^{27}$ and inhibition of the oxidative burst, ${ }^{10}$ are not caused by these toxins but are the result of the action of a novel spore product. Enhanced binding of the $A$ fumigatus spores in inflamed lung ${ }^{28}$ may assist in bringing the spore toxin into close contact with lung cells.

In summary, a small molecular weight toxin $(<10 \mathrm{kD})$ that is released from the spores of a clinical isolate of $A$ fumigatus within minutes of deposition in aqueous solution, which is capable of inhibiting the oxidative burst of macrophages without causing cell death, ${ }^{10}$ is distinct from common fungal toxins, particularly gliotoxin. Further research will be focused on molecular characterisation of the $A$ fumigatus diffusate toxin and elaboration of its effect on intracellular signalling pathways in macrophages that leads to activation of the NADPH oxidase complex and cytokine gene expression. $^{29-31}$

1 Seaton A, Robertson MD. Aspergillus, asthma and amoebae. Lancet 1989:893-4

2 Bateman ED. A new look at the natural history of Aspergillus hypersensitivity in asthmatics. Respir Med 1994;88:325-7. 3 Mullins J, Harvey R, Seaton A. Sources and incidence of airborne Aspergillus fumigatus. Clin Allergy 1976;6:209-17.

Waldorf AR, Lentz SM, Diamond RD. In vivo bronchoalveolar macrophage defence against Rhizopus oryzae an Aspergillus fumigatus. I Infect Dis 1984;150:752-60.

5 Robertson MD, Seaton A, Raeburn A, Milne LJR. Inhibition of phagocyte migration and spreading by spore diffusates of Aspergillus fumigatus. F Med Vet Mycol 1987;25:389-96.

6 Robinson BWS, Venaille TJ, Mende AHW, McAleer R Allergens as proteases: Aspergillus fumigatus protease directly induces human epithelial cell detachment. $\mathcal{F}$ Allergy Clin Immunol 1990;86:726-31

7 Reichard V, Buthner S, Eiffert H, Ruchel R. Purification and characterisation of an extracellular serine protease from Aspergillus fumigatus and its detection in tissue. $\mathcal{F}$ Med Microbiol 1990;33:243-51.

8 Robertson MD, Seaton A, Milne LJR, Raeburn A. Suppression of host defences by Aspergillus fumigatus. F Med Vet Mycol 1987;25:389-96.

9 Robertson MD, Seaton A, Milne LJR, Raeburn A. Resistance of spores of Aspergillus fumigatus to ingestion by sistance of spores of Aspergillus fumigatus

10 Slight J, Nicholson WJ, Mitchell CG, Pouilly N, Beswick $\mathrm{PH}$, Seaton A, et al. Inhibition of the alveolar macrophage oxidative burst by a diffusible component from the surface of the spores of the fungus Aspergillus fumigatus. Thorax 1995;51:389-96.

11 Nicholson WS, Slight J, Donaldson K. Inhibition of cytokine gene transcription by a diffusable component from the spores of the fungus Aspergillus fumigatus; role of the transcription activating factors $\mathrm{NF}-\mathrm{\kappa B}$ and $\mathrm{AP}-1 . A m$ Respir Cell Mol Biol 1996;15:88-96.

12 Waring P, Newcombe N, Edel M, Lin QH, Jiang H, Sjaarda $\mathrm{A}$, et al. Cellular uptake and release of the immunomodulating fungal toxin gliotoxin. Toxicology 1994 32:491-504.

13 Johnson RB, Godzik CA, Cohn ZA. Increased superoxide by immunologically activated and chemically elicited macrophages. $\mathcal{F}$ Exp Med 1978;148:115-27.

14 Richard JL, Lyon RL, Fichtner RE, Ross PF. Use of thinlayer chromatography for detection and high performance liquid chromatography for quantitating gliotoxin from rice cultures of Aspergillus fumigatus Fresenius. Mycopathologia cultures of Aspergillus

15 Ehrlich KC, DeLucca II AJ, Ciegler A. Naphtho- $\gamma$-pyrone production by Aspergillus niger isolated from stored cottonseed. Appl Environ Microbiol 1984;48:1-4.

16 Tanaka H, Wang P-L, Yamada O, Tamura T. Yellow pigments of Aspergillus niger and Asp. awamori. I. Isolation of Aurasperone-A and related pigments. Agric Biol Chem 1972;30:107-13.

17 Cole RJ, Kirksey JW, Dorner JW, Wilson DM, Johnson Jr JC, Johnson AN, et al. Mycotoxins produced by Aspergillus 
fumigatus species isolated from molded silage. 7 Agric Food Chem 1977;25:826-30.

18 Sturtevant JE, Latge J-P. Interactions between conidia of Aspergillus fumigatus and human complement component C3. Infect Immun 1992;60:1913-8.

19 Arruda KL, Mann BJ, Chapman MD. Selective expression of a major allergen and cytotoxin, Asp F 1 in Aspergillus fumigatus: implications for the immunopathogenesis of Aspergillus-related diseases. F Immunol 1992;10:3354-9.


potential. Environ Health Perspect 1986;66:105-8

21 Waring P, Eichener RD, Palni T, Mullbacher A. The isolation and identification of a new metabolite from Aspergillus fumigatus related to gliotoxin. Tetrahedron Lett 1985; 27:735-8.

22 Reijula KE. Monoclonal antibodies bind identically to both spores and hyphae of Aspergillus fumigatus. Clin Exp Allergy 1992;22:547-53.

23 Yamano T, Kishino K, Yamatodani S, Abe M. Researches on ergot fungus. Part XLIX. Investigation on ergot alkaloids occurred in culture of Aspergillus fumigatus Fres. Annu Rep Takeda Res Lab 1962;21:95-101.
24 Moreau C. Moulds, toxins and food. Wiley, 1974

25 Mullbacher A, Waring P, Eichener RD. Identification of an agent in cultures of Aspergillus fumigatus displaying antiphagocytic and immunomodulating activity in vitro. $f$ Gen Microbiol 1985;131:1251-8.

26 Eichner RD, Salami MA, Wood PR, Mullbacher A. The effect of gliotoxin upon macrophage function. Int $\mathcal{f} \mathrm{Im}$ munopharmacol 1986;8:789-97.

27 Brown K, Gerstberger S, Carlson L, Franzoso G, Siebenlist $\mathrm{U}$. Control of IKB-alpha proteolysis by site-specific, signal induced phosphorylation. Science 1995;267:1485-9.

28 Bromley IMJ, Donaldson K. Binding of Aspergillus fumigatus spores to lung epithelial cells and basement membrane proteins: relevance to the asthmatic lung. Thorax 1996 51:1203-9

29 Mullbacher A, Eichner RD. Immunosuppression in vitro by a metabolite of a human pathogenic fungus. Proc Nat Acad Sci USA 1984;81:3835-7.

30 Cross A. Inhibitors of the leucocyte superoxide generating system. Free Radic Biol Med 1990;8:71-93.

31 Bastian NR, Hibbs JB. Assembly and regulation of NADPH oxidase and NO synthase. Curr Opin Biol 1994;6:131-3. 